\title{
Lightness contrast in CRT and paper-and-illuminant displays
}

\author{
TIZIANO AGOSTINI and NICOLA BRUNO \\ University of Trieste, Trieste, Italy
}

\begin{abstract}
The increased use of CRT monitors for displaying and controlling stimuli in studies of surface color poses problems of comparability with data obtained with traditional paper-and-illuminant methods. A review of comparable studies using the two methodologies revealed that CRT studies tend to report larger contrast effects. To investigate factors that may be responsible for this difference, simultaneous lightness contrast was measured using both CRT and paper-and-illuminant presentations. The spatial distribution of luminance in the whole field of view and the visual angles subtended by the displays were controlled. The CRT presentation yielded contrast effects twice as big as those measured for a paper surface in a homogeneously illuminated room. However, a paper display under Gelb lighting yielded almost exactly the same effect size as that measured in the CRT presentation. These results demonstrate that contrast effects in both modes of presentation are affected by the spatial distribution of luminance beyond the basic experimental stimuli.
\end{abstract}

Increasingly, investigators of achromatic surface color (lightness) have been reporting experiments based on judgments of surfaces simulated on CRT monitors (Agostini \& Proffitt, 1993; Arend \& Goldstein, 1987a, 1987b; Arend \& Reeves, 1986; Arend \& Spehar, 1993a, 1993b; Bruno, 1994; Kingdom \& Moulden, 1991; Paradiso \& Nakayama, 1991; Schirillo, Reeves, \& Arend, 1990; Schirillo \& Shevell, 1993a, 1993b; Shevell, Holliday, \& Whittle, 1992; Whittle, 1992). Using computer simulations involves careful control of the spatial distribution of monitor intensities to evoke visual responses equivalent to those evoked by surfaces and illuminant. However, perfect equivalence is hard to achieve in practice. This difficulty has consequences for the comparability of CRT data with data obtained with paper-andilluminant methods. What differences in the overall intensity distributions can be allowed without affecting comparability? What characteristics of CRT monitors and of paper-and-illuminant displays make it difficult to equate corresponding spatial distributions of luminance? These are empirical questions, and answers to them are critical to the development of the field. Yet, to this date no published work has addressed them. In this paper, a first step is made in this direction by measuring one of the most basic effects of lightness perception, simultaneous contrast, in several comparable CRT and paper-andilluminant displays.

The authors are indebted to M. Braunstein, S. Shevell, and two anonymous reviewers for helpful comments on an earlier draft, and to A. Gilchrist for suggesting that we perform the present experiments. This work was supported in part by CNR Grant 92.01065.PF67. Correspondence should be addressed to T. Agostini, Department of Psychology, University of Trieste, via Universitá 7, 34123 Trieste, Italy (e-mail: agostini@univ.trieste.it).
In simultaneous lightness contrast (SLC), two achromatic surfaces are presented side by side on black-andwhite backgrounds. The surfaces have the same reflectance, but they appear to be different. The surface surrounded by black appears to be lighter; that surrounded by white, darker. In most studies of contrast, the size of this difference (the contrast effect) is reportedly rather small. A number of paper-and-illuminant studies of the standard contrast arrangement have reported effects on the order of about 1 Munsell unit (Gibbs \& Lawson, 1974; Gilchrist, 1988; Gilchrist, Delman, \& Jacobsen, 1983; White, 1981). In studies of contrast involving a greater degree of spatial articulation, even weaker effects have sometimes been reported. ${ }^{1}$ For instance, Agostini and Gerbino (1989) placed middle gray surfaces in a context made of disks on a homogeneous background. The disks were either white on a black background or black on a white background. The whole display was mounted on a curved surface and placed in a viewing box. Observers viewed it through an aperture so that the display filled their field of view. Observed contrast effects were on the order of 0.5 Munsell units. Only a few paper-and-illuminant studies reported somewhat larger contrast effects. For instance, Burgh and Grindley (1962) found average contrast effects of about 2 Munsell units in the traditional bipartite arrangement.

How do these results compare to CRT measures of contrast? A first answer may be found in CRT studies that can be compared to corresponding paper-andilluminant studies either because they used displays that were similar spatially or because of comparable levels of articulation. For instance, Bruno (1994) replicated previous paper-and-illuminant experiments by Gilchrist (1988) using a CRT simulation. The CRT display was equivalent to Gilchrist's in terms of luminances and spatial relationships, but it subtended a smaller visual angle 
than Gilchrist's and it was observed in an otherwise dark room. Bruno found contrast effects as large as 2.5 Munsell units under conditions comparable to those where Gilchrist found effects of about 1 unit. In a number of CRT studies, Arend and his collaborators placed centersurround displays similar to those used to demonstrate SLC within the context of larger achromatic Mondrians (for a summary of all these studies, see Arend, 1994). Typical displays for these studies did not fill the entire field of view. However, because of the spatial articulation provided by the Mondrian surround, they could be taken as somewhat comparable to those of Agostini and Gerbino (1989). Arend reported overall deviations from correct Munsell matches to be of the order of 1.0-1.5 units. In conclusion, it seems that CRT studies of contrast report somewhat larger effects than comparable paper-and-illuminant studies. Candidate explanations for this unexpected difference are naturally grouped into three categories.

The first category consists of factors that cannot be separated in principle from the method of presentation. For instance, a monitor is a light-emitting device, whereas surfaces reflect light from the illuminant. However, this difference should be irrelevant as long as the spatial distribution of light at the eye is kept the same. More concretely, most monitors have intensity ranges of about 2 logarithmic units and resolution of 256 discrete gray levels. It is possible that range, small imperfections in monitor calibration, or both, contribute in some unknown way to increasing contrast effects. With paper-andilluminant displays, on the other hand, it is often quite hard to achieve complete control of the spatial distribution of the illumination and to measure it accurately. Unwanted and unnoticed spatial variation of the illumination, or failures to measure it to a satisfactory degree of accuracy, may also affect measures of contrast in some unknown way. However, the effect of these factors should not be systematic across different studies.

The second category consists of factors that are readily separated from the method of presentation, but tend to be correlated with it for reasons of convenience. For instance, monitor studies often tend to employ displays that subtend smaller visual angles than do displays used in paper-and-illuminant studies. Given that edge distances are known to have a small but measurable effect (Freeman, 1967; Jund \& Armington, 1975), it could be that this provokes a detectable increase in contrast. It may be that monitor outputs suffer from imperfections in calibration, or display luminances on the monitor may be affected by veiling light and reflections off the monitor glass, or pixel texture may alter actual luminances on the screen. It might be that subtle effects of this kind are responsible for the difference. However, it seems unlikely that the discrepancy in data obtained with the two methods is due to these factors. Most investigators are well aware of them, and the reviewed studies carefully controlled for them.

Finally, the third category consists of factors that are separable in principle from the method of presentation, but are difficult to separate in practice. Of chief interest are the observation conditions for a monitor display. In typical studies, CRT-simulated displays are presented in a dark or dimly lit room. Thus, the monitor becomes an area of relatively high mean luminance, surrounded by darkness or near darkness. Having the monitor as the only source of light in the room is desirable for a number of reasons. First of all, turning off other sources of illumination eliminates the possibility of veiling light, allowing more precise control of display luminances. Second, it prevents reflections from forming on the screen, again allowing better control of luminance and increasing display realism. On the other hand, in many paper-and-illuminant studies, observers are able to see both the experimental display and the surfaces surrounding it. Thus, once the whole field of view is taken into account, paper-and-illuminant displays may involve a rather different overall spatial distribution of luminance than CRT displays even if the luminances of the SLC stimulus are carefully equated.

There are reasons to suspect that observation conditions may be an important factor in accounting for the observed difference in SLC effects between CRT and paper-and-illuminant displays. For one, it is instructive that Burgh and Grindley (1962) reported unusually large contrast effects despite their using a paper-and-illuminant method. In their experimental setup, the display was surrounded by an otherwise dark field so that their observation conditions closely mimicked those that are typical of CRT studies. Another factor may be lightness effects observed on surfaces placed under a strong beam of light but otherwise surrounded by darkness. Gelb (1929) showed that a black surface appears white if it is illuminated in such a way that the border of the illumination coincides with the border of the surface and the rest of the field is in darkness. That the surface appears white is perhaps not too surprising, for the system has information concerning only the relationship of this area of high luminance to the rest of the field, which is dark. However, anybody who has tried demonstrating the Gelb effect knows that these conditions are rarely attained in full. In most cases, one is able to cast a strong beam of light on a black surface suspended in front of a wall, but the wall is also illuminated in part. Even in these conditions, however, the black surface appears almost white to most observers, a fact consistent with the speculation that the illumination border somehow prevents placing the surface in the appropriate relationships to the other luminances in the field of view.

\section{EXPERIMENT 1}

The first experiment consisted of a basic comparison between the two methods of presentation. Two sets of displays were created that had the same spatial distribution of luminances and subtended the same visual angle. The CRT displays were observed using the customary procedure - that is, with the monitor in an otherwise dark room. The surface displays were illuminated by a beam 
adjusted in such a way that the border of the beam coincided with the outside edge of the SLC stimulus. We call this manipulation Gelb lighting, by analogy with the Gelb effect. By placing the surface displays under Gelb lighting in both methods of presentation, we were able to present the SLC stimuli as areas of relatively high luminance surrounded by near darkness.

\section{Method}

Observers. Forty-eight observers volunteered. They were all undergraduate or graduate students of the University of Trieste who were naive to the rationale of the study.

Proximal stimuli. Spatial distributions of luminances are illustrated in Figure 1. Four versions of the standard SLC display were constructed by combining two large rectangular areas $\left(3.5^{\circ} \times\right.$ $5^{\circ}$ of visual angle) and presenting them side by side. One of these was always the highest luminance $\left(75 \mathrm{~cd} / \mathrm{m}^{2}\right)$ and appeared white. The other had a luminance of $1.5,11,23$, or $75 \mathrm{~cd} / \mathrm{m}^{2}$. Centered on this second area was a smaller square area (the target), which had a constant luminance of $16 \mathrm{~cd} / \mathrm{m}^{2}$ in all displays. Centered on the highest luminance area was a set of 16 smaller rectangles, graded in luminance so as to encompass the whole Munsell scale, from black (2.0/) to white (9.5/). Thus, in two of the four displays the target was an increment relative to its surround. In the other two, the target was a decrement. In one of the decrements, the surface surrounding the target and that surrounding the matching scale were actually the same surface (see Figure 1). These displays served as a control condition, as explained later.

Distal stimuli (equipment and conditions). In the Gelb condition, the displays consisted of Color-Aid papers mounted on cardboard and then carefully compared with the Munsell scale to obtain the best possible approximation to their Munsell value. Approxi-

LUMINANCES $\left(\mathrm{cd} \mathrm{m}^{-2}\right) \quad$ MUNSELL VALUES

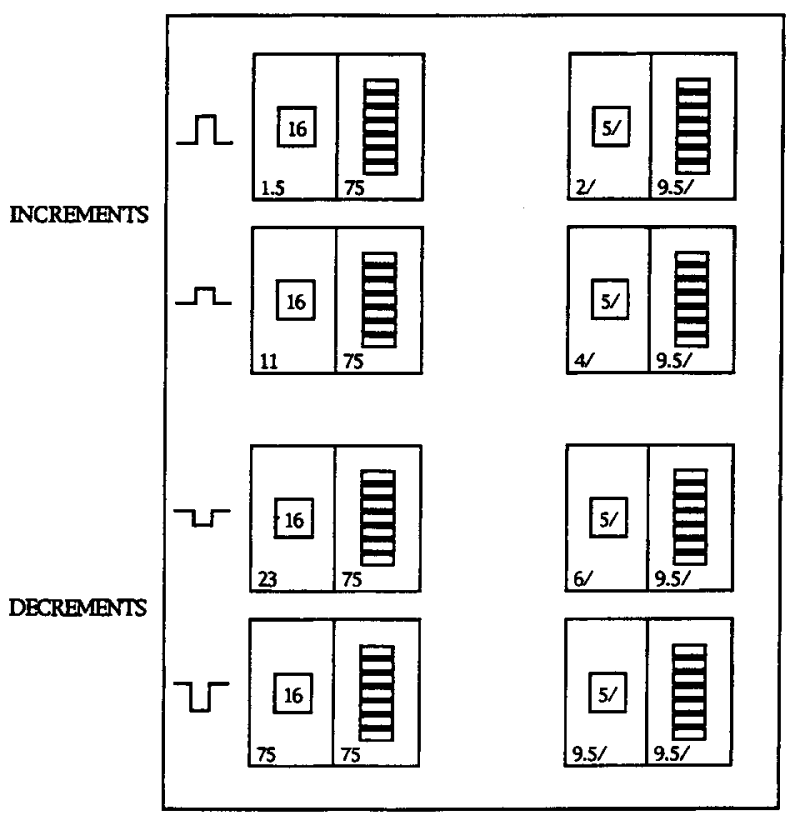

Figure 1. Spatial distribution of luminances in the basic simultaneous lightness contrast displays used in the experiments. Corresponding Munsell values refer either to actual values of paper displays or to simulated values on CRT displays. The bottom decrement is a control display where no effect is predicted. mate Munsell values of these papers (Figure 1) were 9.5/ for the highest luminance surround, 2.0/, 4.0/, and 6.0/ for the other surrounds, and 5.0/ for the target. The Munsell scale that was placed on the highest luminance surround was made of actual Munsell papers. The displays were mounted on a white wall and seen from $2 \mathrm{~m}$. A rectangular beam of light from an adjustable halogen lamp was cast on the wall so that its illumination edges coincided exactly with the outer border of the display. This condition was run in a large room dimly illuminated by indirect light that also originated from the halogen lamp.

In the CRT condition, the displays consisted of luminous areas presented on a carefully calibrated Silicon Graphics RGB monitor (256 gamma-corrected gray values, $1280 \times 1024$ pixels, $60 \mathrm{~Hz}$ refresh rate). Monitor calibration was performed in two steps. First, we obtained photometer readings of the darkest and brightest grays that could be produced on the monitor and adjusted the cont rast and brightness switches to bring them into the appropriate range. Next, we measured luminances at different areas of the monitor and at different gray values, and we then fitted appropriate polynomials to obtain the equation for converting software-specified intensities to the desired luminances. The luminance, spatial characteristics, and the viewing distance of these areas were controlled so that the resulting proximal stimuli were the same as those of the Gelb condition. That is, the whole screen was divided vertically into two areas that simulated the background luminances of the paper display. In this way, the simulated display was surrounded by the frame of the screen, which was hardly visible. As in the former condition, these CRT-simulated surfaces were observed in a room that was dimly illuminated by indirect light originating from the monitor.

Procedure. Observers waited in a normally illuminated area; they walked into the room and were immediately taken in front of the white wall (Gelb condition) or seated in front of the monitor (CRT condition) at the appropriate distances. After they had seen the display, the experimenter read the following instructions while pointing to the corresponding surfaces: "In front of you is a square paper attached to the center of a rectangular cardboard. Next to it is a series of rectangular gray patches ranging from white to black. Your job is to identify the patch that has been cut from the same gray paper as the square paper." Observers responded by pointing to one of the patches and then left the room. Each observer performed one match in one condition only.

\section{Results}

Figure 2 illustrates median contrast effects and distributions of matches in the two conditions. Contrast effects were measured as differences in Munsell units between the median match in the control displays (where no contrast effect was expected) and observer matches in the other three displays. Here as well as in the second experiment, we chose to measure contrast effects relative to the control condition because, as stated above, the paper target was not a Munsell paper, and its exact Munsell value could only be approximated. In fact, the median Munsell match for the target in the Gelb condition control, at least in the present observation conditions, turned out to be 5.5/. As can be seen from the histograms, contrast effects were almost exactly identical with both methods of presentation. Additionally, they were much larger than those reported in most paper-and-illuminant studies. Median contrast effects were 2.75/ Munsell units for all CRT and Gelb increments, 1.75/ for the CRT decrement, and 2.5/ for the Gelb decrement. The difference between decrements was significant (Mann-Whitney $U=$ $.5, p<.01)$. 


\section{CRT in a dark room Paper under GELB lighting}

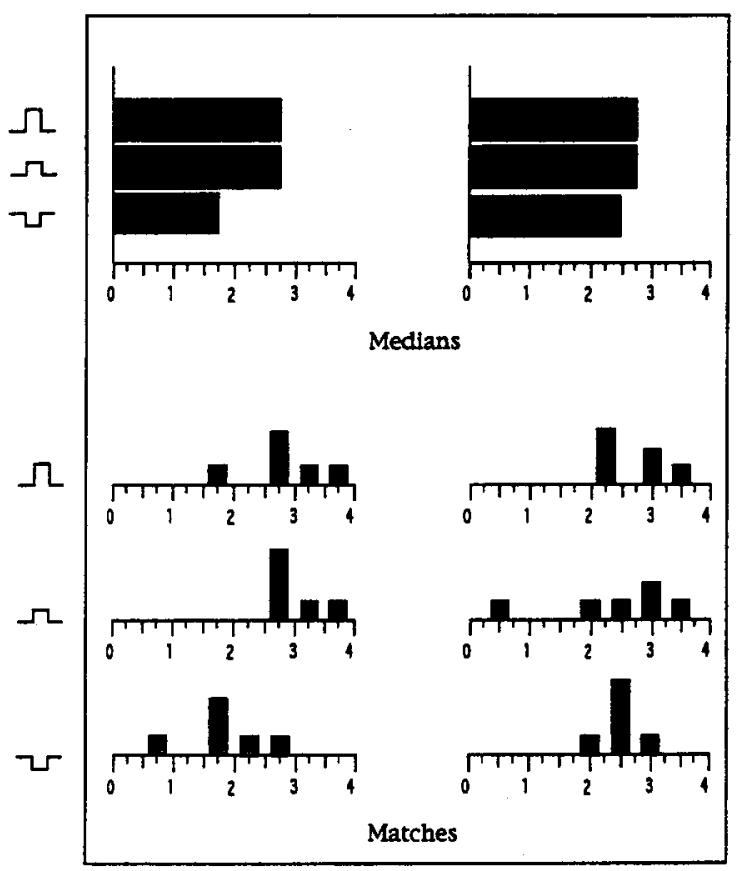

Contrast effect (Munsell units)

Figure 2. Median contrast (top) and distributions of effects (bottom) in Experiment 1 for the larger increment, the smaller increment, and the decrement displays. Contrast effects were measured as differences, in Munsell units, between individual Munsell matches for a given display and the corresponding median match for the control decrement.

\section{Discussion}

These results suggest that CRT estimates of contrast may be biased by the observation conditions that are typical of CRT studies. When these are reproduced in a paperand-illuminant display, the size of the contrast effect is increased in a fashion that is very similar to what is observed in a CRT display.

\section{EXPERIMENT 2}

If the different behavior of contrast on a monitor display is due to the fact that the monitor is an area of higher luminance within a dimmer context, it should be possible to control the size of the contrast effect in paper-andilluminant displays by including progressively more of the context within the same area of higher mean luminance. To test this possibility, we performed a second experiment. Starting from the Gelb condition of Experiment 1 , where the illumination edge coincided with the outer border of the SLC stimulus, we first enlarged the size of the beam so as to include part of the context within the same illumination edge. As a result of this manipulation, a larger area of the field of view was perceived under the same framework of illumination. Then, we repeated our measurements using two kinds of approx- imately homogeneous illuminations for the whole field of view. In these two latter cases, the entire field of view was perceived as being under the same illumination.

\section{Method}

Observers. An additional 72 observers volunteered. They were all undergraduate or graduate students of the University of Trieste who were naive to the rationale of the study and who had not participated in the first experiment.

Stimuli, Apparatus, Procedure, and Design. The method was the same as that of Experiment 1, with the following changes. First, in this experiment we used only paper surfaces. Second, we manipulated the spatial modulation of the illumination as well as the visibility of the surrounding context. More precisely, we measured contrast effects in three different conditions. In the enlarged condition, the beam of light cast on the displays was changed so as to illuminate a circular area on the wall (diameter $=30^{\circ}$ of visual angle) centered about the displays. In the homogeneous condition, the illumination was modified so that the whole room was illuminated by an approximately homogeneous illumination. Finally, in the daylight condition, the displays were simply placed in a small garden outside our laboratory on a moderately sunny day. Except for the change in the surrounding context, the luminances and the spatial characteristics of the displays were exactly the same as those in the previous experiment. Note, however, that this was not true in the daylight condition, where we had no control over the illumination.

\section{Results}

Figure 3 illustrates median contrast effects and distributions of matches in the three new conditions. The distribution of effects in the Gelb condition of the previous experiment is also plotted for comparison. As in the previous experiment, contrast effects were measured as differences relative to median matches in the control displays, which were 5.25/ in the enlarged and in the homogeneous conditions, and 5.75/ in the daylight condition.

Relative to the Gelb condition of the previous experiment, in the homogeneous condition, contrast effects were reduced from 2.75/ to 0.75 / Munsell units for increments and from $2.5 /$ to $0.25 /$ for the decrement. All these differences were significant (Mann-Whitney $U<4$ for all three displays; $p<.02$ or smaller). In the enlarged condition, contrast was also reduced, from $2.75 /$ to 1.75 / for increments and from $2.5 /$ to 0.25 / for the decrement. These differences were also significant $(U<1.5 ; p<$ .01 ), except for the smaller increment, which failed to reach significance $(U=8, p>.1)$. Contrast effects in the daylight condition were only slightly larger than those in the homogeneous condition. They were $1.0 /$ and $1.5 /$ units for the larger and the smaller increments, respectively, and $0.75 /$ units for the decrement.

\section{Discussion}

These results confirm that the size of the contrast effect is due to the influence of the luminances outside of the SLC stimulus. As a larger portion of the field of view is included within the same framework of illumination as the SLC stimulus, the contrast effect is reduced. Contrast effects obtained in the homogeneous condition are comparable to those found in typical paper-and- 


\section{PAPER}

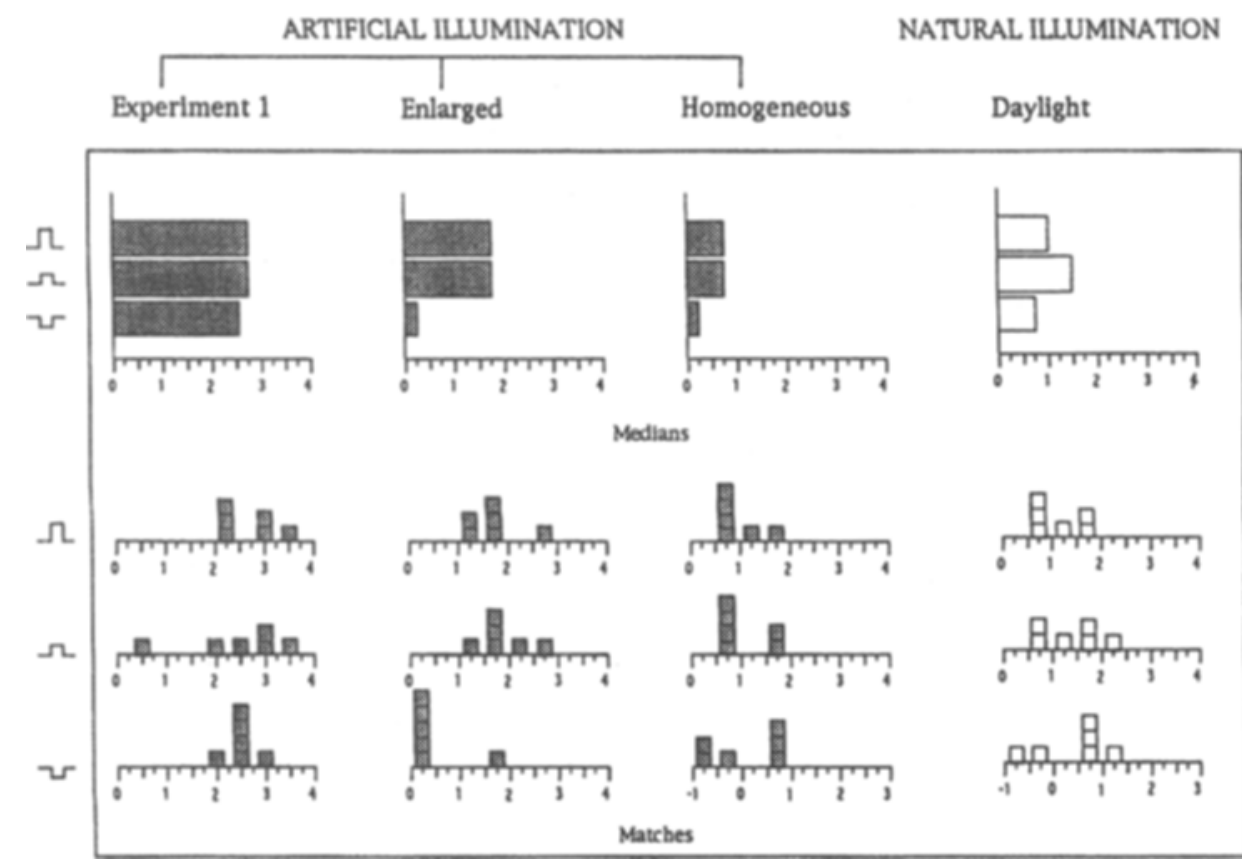

Contrast effect (Munsell units)

Figure 3. Median contrast and distributions of effects in Experiment 2. Layout of histograms and bar charts as in Figure 2. As in Experiment 1, contrast effects were measured as differences, in Munsell units, between individual Munsell matches for a given display and the corresponding median match for the control decrement.

illuminant experiments. In the daylight condition, which should be the most ecologically valid, the size of contrast was slightly larger than in the homogeneous condition. This is likely due to uncontrolled variations in the spatial distribution of luminance during slight changes in sunlight during the day. Presumably, repeated measures over different days would converge to the same value we obtained in the homogeneous condition. Thus, results from these two conditions provide the best approximation to the size of contrast under natural viewing conditions. Relative to these conditions, contrast increased as the spatial distribution of illumination included less of the context. This finding suggests a way of controlling and maybe reducing contrast effects in CRT-simulated displays.

\section{EXPERIMENT 3}

The aim of the third experiment was to test whether contrast effects on CRT-simulated displays can be reduced by providing a larger context. One might think that this could be done just by turning on the light in the laboratory so that the larger context becomes visible. However, this solution has two serious problems. The first concerns reflections. Light reflected off the glass screen would create a veiling luminance on the display and possibly areas of highlight. This would hamper control of display luminances. The second concerns the apparent distribution of the illumination. Consider, for instance, what would happen if one tried to provide a larger context to the monitor by surrounding it with a surface. In the typical viewing conditions for a monitor study, the surface would not be illuminated, and the monitor would still be an area of high luminance surrounded by darkness. If the surface were illuminated, on the other hand, it would be difficult to equate the intensity and spectral composition of this added illumination to the simulated illumination on the monitor display. Given these practical difficulties, we decided to keep the monitor as the only source of light in the room and to explore instead the effect of providing a context within the monitor itself. This strategy has been already used in some CRT studies (see Arend, 1994), but has never been investigated in a direct fashion.

\section{Method}

Observers. An additional 36 observers volunteered. They were all undergraduate or graduate students of the University of Trieste who were naive to the rationale of the study and who had not participated in the first two experiments.

Stimuli, Apparatus, and Procedure. The method was the same as that of Experiment 1, with two exceptions. First, in this experiment we only used displays simulated on a CRT. Second, we ma- 
nipulated the spatial characteristics of these displays as well as the simulated context surrounding them. More precisely, we measured contrast effects in three different conditions. In the small CRT condition, we applied a black cardboard mask on the monitor so that it covered the screen. This mask had a rectangular hole in the middle $\left(5^{\circ} \times 2^{\circ}\right)$. Through this hole, observers could see the same SLC display that we had used in the first experiment, rescaled in size to fit the portion of the monitor that could be seen through the hole. This condition served as a baseline measurement to control for the change in visual angle relative to the CRT display of Experiment 1. In the gray surround condition, we removed the cardboard mask and surrounded the rescaled SLC display with a homogeneous field, which appeared middle gray $\left(16 \mathrm{~cd} / \mathrm{m}^{2}\right)$. Finally, in the Mondrian surround condition, we replaced the gray field with a Mondrian pattern. The luminances of the Mondrian were random samples with replacement from a population of equally probable luminances $\left(1<\mathrm{L}<75 \mathrm{~cd} / \mathrm{m}^{2}\right)$.

Design. The experimental design was similar to that of the first two experiments. However, to further simplify it we dropped the higher increment, which yielded essentially the same magnitude of contrast as the second increment. For the same reason, we also dropped the control display, which served the purpose of controlling for approximation errors in estimating Munsell values, and was therefore not necessary on a CRT. Additionally, each observer also performed two separate matches. One was the familiar match with the simulated Munsell scale, which was surrounded by a white area and was presented on the side of the area surrounding the target. The other was a match with an actual Munsell scale, having the same number of steps and the same range as the simulated scale. The actual Munsell scale was placed on the floor below the table supporting the monitor. It was illuminated by a lamp placed on top of it so as to be invisible to the observer. The order of these matches was counterbalanced across observers.

\section{Results}

Distributions of contrast effects and median matches are illustrated in Figure 4 for both the simulated and actual Munsell scales. As stated earlier, contrast effects in this experiment were measured as deviations of matches relative to the simulated Munsell value of the target.

Consider first matches using the CRT-simulated scale. Relative to the small CRT baseline, only the Mondrian surround condition yielded smaller median effects for both increment and decrement displays. However, this

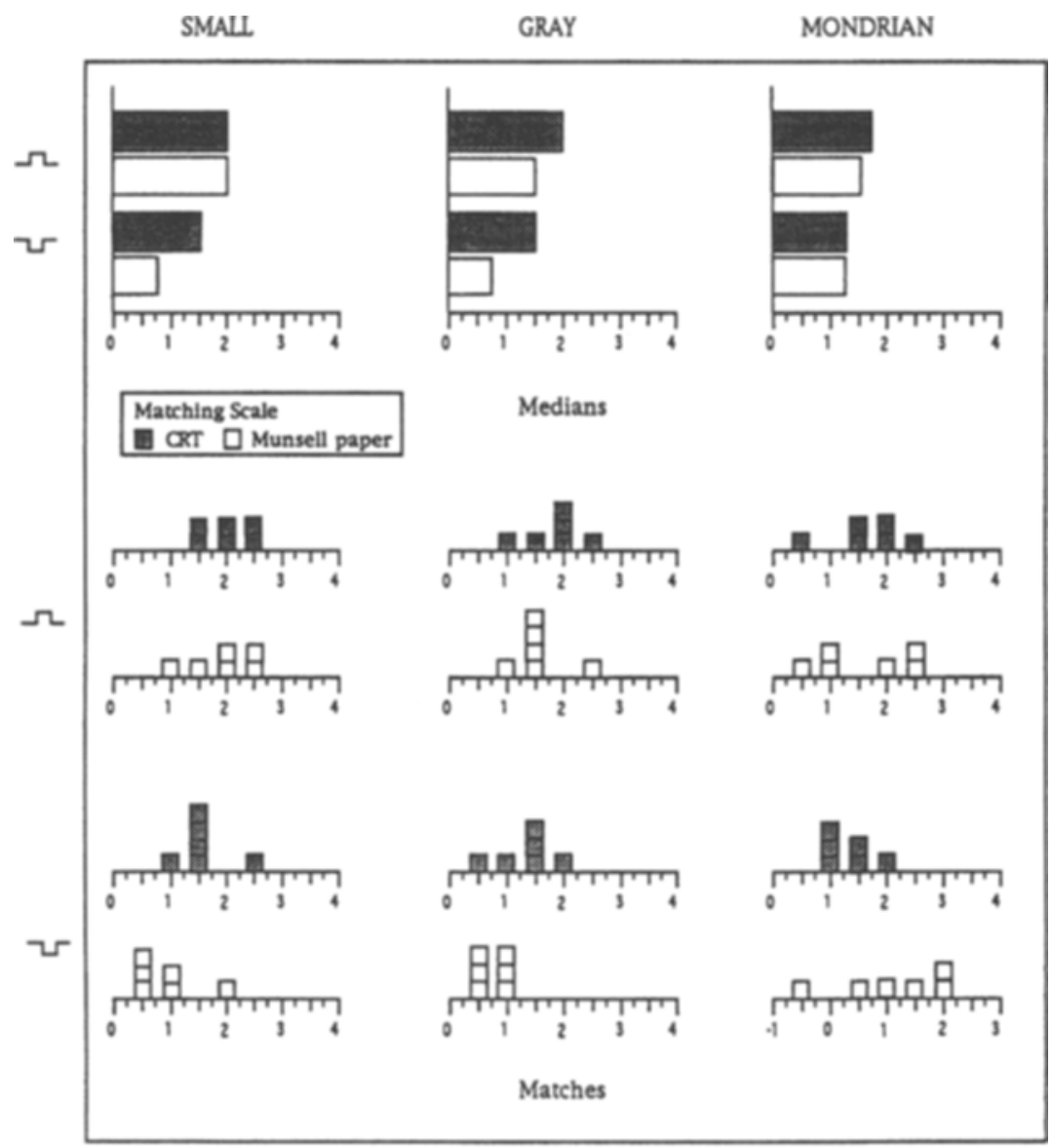

Contrast effect (Munsell units)

Figure 4. Median contrast and distributions of effects in Experiment 3 for the increment and decrement displays and using either a simulated or actual Munsell matching scale . Contrast effects were measured as differences, in Munsell units, between individual matches for a given display and the corresponding simulated Munsell value. 
reduction was small relative to the variability between observers, and failed to reach significance $(U=13$ and $12.5, p>.4$ and .3 for the increment and the decrement, respectively). The median values of the small CRT and gray surround conditions were identical. Next, consider matches using the Munsell paper scale. Relative to the small CRT baseline, the gray surround data showed a smaller median contrast for the increment, but not for the decrement; the former difference failed to reach significance $(U=11.5, p>.2)$. The Mondrian surround condition showed a smaller median contrast for increments, but a greater median contrast for decrements. Again, however, no difference was statistically significant $(U=14$ and $14.5, p \mathrm{~s}>.5$, for increments and decrements, respectively). Overall, median contrast effects were also consistently smaller when measured using the actual Munsell scale than when matching to the simulated scale on the CRT. However, this difference was significant only for decremental displays (Wilcoxon signed rank test, $Z=-2.844, p<.01$ ). In the case of increments, the difference failed to reach significance $(Z=$ $-.847, p>.3$ ).

\section{Discussion}

Contrast measured on a monitor may be affected by a context surrounding the simulated SLC stimulus. However, this effect of context appears to be rather weak and it fails to reach statistical significance. Our failure to find significant differences in this study is likely due to our small sample size and consequent inadequate power. However, further work is certainly needed to determine if this speculation is correct. Given the results of Experiments 1 and 2, it was to be expected that the inclusion of a larger context would contribute to reduce the contrast effect in the CRT simulation. However, three other features of the present results were not predictable from the previous experiments. First of all, it is noteworthy that even a background possessing a high degree of articulation fails to reduce contrast to the levels that are found when the whole field of view appears to be within the same framework of illumination. Based on our results from the homogeneous and daylight conditions of Experiment 2, these seem to be on the order of 0.75-1 Munsell units for increments and of 0.25-0.75 units for decrements, about 1 unit smaller than corresponding contrast effects in the Mondrian surround condition of the present experiment. Therefore, this result seems to indicate that stimulation from the peripheral, and uncontrolled, visual field has a measurable effect on the contrast even when the SLC stimulus is surrounded by a highly articulated field. A second unexpected result is that contrast was actually reduced in the small CRT condition relative to the CRT condition of Experiment 1. Based on earlier results on the effect of visual angle (Freeman, 1967) and of relative edge distance (Jund \& Armington, 1975), one would have expected the opposite outcome, or no difference. Given the small sample size employed in this study, however, we are not in a position to speculate on this result. Finally, also unexpected is the finding that there is a reliable difference between matches to increments, but not to decrements, when using a CRTsimulated scale as opposed to using an actual Munsell paper scale. A possible interpretation for this difference may be found by noting that the actual Munsell scale was placed under a different illumination than that simulated on the monitor. Therefore, when performing matches to the actual Munsell scale, observers were really matching surfaces in front of different backgrounds and also under different illuminations. This might have introduced a different source of variation in lightness matches. In fact, it is known that when two identical surfaces are placed under two different illuminations, lightness constancy shows a partial failure, and this failure is larger for increments than for decrements (Jacobsen \& Gilchrist, 1988). Perhaps failures of constancy under two perceived illuminations for the monitor and the actual Munsell scale interacted in some way with the effect of contrast, and this caused the different behavior of increments and decrements.

\section{GENERAL DISCUSSION}

The present results suggest that a major factor affecting the size of the SLC effect in both CRT and paperand-illuminant displays is the spatial distribution of luminances beyond the basic experimental stimuli. However, in a CRT display the spatial distribution of luminance can be easily controlled only within the monitor itself. Possible interactions between luminances on the monitor and those on the rest of the visual field are not controlled. On the other hand, satisfactory control of all luminances in the whole field is more easily achieved by a paper-andilluminant method. However, even paper-and-illuminant studies of contrast often neglect to measure and report luminances beyond the basic stimuli. For instance, a quick perusal of lightness data published in Perception \& Psychophysics between 1970 and 1990 revealed that, out of 32 published papers, only 14 reported measurements of luminances beyond the basic stimuli, although some reported that the surround of the basic stimuli was covered with black velvet or painted black. Only 13 provided enough information to determine if the surround filled the entire field of view. Several did not describe the arrangement in sufficient detail to speculate as to whether the surround appeared to be under the same framework of illumination as the basic stimulus. Thus, investigators using both methods should be made aware of the need to measure and control all the luminances in the visual field.

Whether this can be achieved satisfactorily in a CRT presentation remains to be determined. In this paper we explored two compromise solutions, based on surrounding basic CRT stimuli with a gray surround or with a richly articulated background. The first manipulation seems to have been only marginally successful in bringing down the size of measured contrast effects to values more similar to those observed when the basic stimuli are in the same framework of illumination as the rest of the visual field. This limited degree of success might be 
due to an inadequate choice of the luminance for the gray surround. According to some reports, a gray surround equal to the spatially weighted average of a chromatic Mondrian has the same effect as that of the Mondrian itself on the chromaticity of a patch (Valberg \& Lange-Malecki, 1990). Given that our gray surround was not a spatially weighted average of the Mondrian (although it was not too far from it), it is possible that selecting the appropriate value would have caused the gray surround to behave more like our second explored compromise, which involved precisely a Mondrian. In other studies, however, the notion of an "equivalent background" has proved of limited value in predicting lightness matches (Bruno, 1992; Schirillo \& Shevell, 1993a, 1993b). The second compromise solution, used in several published works by Arend and his collaborators (see Arend, 1994), yielded a trend in the direction of a reduction of observed contrast. However, this trend failed to reach significance and a substantial difference remained. This should be taken into consideration if the absolute size of contrast is of theoretical concern.

Although the present results were quite clear in identifying an often uncontrolled factor affecting the size of SLC, a number of other questions remain. One concerns the explanation of the effect of Gelb viewing conditions. It is possible, for instance, that the change in the size of contrast is due to interactions between receptor gain controls as the retinal periphery is put in conditions of near dark adaptation. However, preliminary observations from another laboratory (A. Gilchrist, personal communication, September 1994) suggest that a similar change in the size of contrast is observed even if the SLC stimulus is placed under a shadow in an otherwise highly illuminated room, a condition that might be called Kardos lighting (Kardos, 1934). Another possibility is that the critical factor is not surrounding darkness per se, but the fact that the basic SLC stimulus and the dark (or light) surround are perceived to lie under different frameworks of illumination. Recent work by Cataliotti and Gilchrist (1995) suggests that edge integration within local and global frameworks may indeed be a critical factor in determining the outcome of lightness computations in Mondrian-like scenes. Given that the present experiments were not designed to address mechanisms responsible for the effect of Gelb lighting, however, we are not in a position to reach any final conclusion on this issue.

A second unanswered question concerns the relevance of the present results to studies of surface color constancy under CRT-simulated spatial variations of the illumination. A number of studies have looked at this question using a CRT methodology (Arend \& Spehar, 1993a; Arend \& Goldstein, 1987a, 1987b; Bruno, 1994; Schirillo et al., 1990; Schirillo \& Shevell, 1993a, 1993b). Several other studies have used CRT displays to study a similar question with chromatic surface color (Arend, 1993; Arend \& Reeves, 1986; Arend, Reeves, Schirillo, \& Goldstein, 1991; Craven \& Foster, 1992; Foster, Craven, \& Sale, 1992; Lucassen \& Walraven, 1993). None of these studies controlled the intensity and the spectral compo- sition of light from the visual field surrounding the monitor. It is possible, therefore, that these measurements were also affected to some extent by those uncontrolled luminances. However, in a preliminary study of failures of lightness constancy under variations of real and CRTsimulated illuminations, we failed to find significant differences in pattern between paper and CRT displays. Additionally, during the preparation of this paper we became aware of work on this issue by O'Shea and Savoy (O'Shea \& Savoy, 1995; Savoy \& O'Shea, 1993). In a careful comparison of chromatic constancy under spatial variations of the illumination, they found essentially no differences between the two methods of presentation. It is possible, therefore, that factors affecting contrast on CRT simulations do not equally affect measures of lightness or color constancy under variations of the illumination, perhaps because simulating two or more differently illuminated areas on a CRT forcibly requires the introduction of a degree of articulation in the simulated display. Further parametric work is needed to establish if this is true in general.

\section{REFERENCES}

Agostini, T., \& Gerbino, W. (1989). Brillantezza della figura, contrasto simultaneo e invarianza colore/illuminazione [Figure brightness, simultaneous contrast, and colour/illumination invariance]. Atti del VIII Congresso Nazionale della Divisione Ricerca di Base in Psicologia, Trieste.

Agostini, T., \& Proffitt, D. R. (1993). Perceptual organization evokes lightness contrast. Perception, 22, 263-272.

AREND, L. E. (1993). How much does illuminant color affect unattributed colors? Journal of the Optical Society of America A, 10, 21342147.

AREND, L. E. (1994). Surface colors, illumination, and surface geometry: Intrinsic-image models of human color perception. In A. Gilchrist (Ed.), Lightness, brightness, and transparency (pp. 159213). Hillsdale, NJ: Erlbaum.

AREND, L. E., \& Goldstein, R. (1987a). Lightness models, gradient illusions, and curl. Perception \& Psychophysics, 42, 65-80.

Arend, L. E., \& Goldstein, R. (1987b). Simultaneous constancy, lightness, and brightness. Journal of the Optical Society of America $A, 4,2281-2285$.

AREND, L. E., \& ReEves, A. (1986). Simultaneous color constancy. Journal of the Optical Society of America A, 3, 1743-1751.

Arend, L. E., Reeves, A., Schirillo, J., \& Goldstein, R. (1991). Simultaneous color constancy: Papers with diverse Munsell values. Journal of the Optical Society of America A, 8, 661-672.

AREND, L. E., \& SPEHAR, B. (1993a). Lightness, brightness, and brightness contrast: 1. Illuminance variation. Perception \& Psychophysics, 54, 446-456.

AREND, L. E., \& SPEHAR, B. (1993b). Lightness, brightness, and brightness contrast: 2 . Reflectance variation. Perception \& Psychophysics, 54, 457-468.

Bruno, N. (1992). Lightness, equivalent backgrounds, and the spatial integration of luminance. Perception, 21(Suppl.), 80.

Bruno, N. (1994). Failures of lightness constancy, edge integration, and local edge enhancement. Vision Research, 34, 2205-2214.

Burgh, P., \& Grindley, G. C. (1962). Size of test patch and simultaneous contrast. Quarterly Journal of Experimental Psychology, 14, 89-93.

Cataliotti, J., \& Gilchrist, A. (1995). Local and global processes in surface lightness perception. Perception \& Psychophysics, 57, 125-135.

Craven, B. J., \& Foster, D. H. (1992). An operational approach to colour constancy. Vision Research, 32, 1359-1366.

Foster. D. H., Craven, B. J., \& Sale, E. R. H. (1992). Immediate colour constancy. Ophthalmic \& Physiological Optics, 12, 157-160. 
Freeman, R. B. (1967). Contrast interpretation of brightness constancy. Psychological Bulletin, 67, 165-187.

GeLB, A. (1929). Die "Farbenkostanz" der Sehdinge [Object color constancy]. In W. A. von Bethe (Ed.), Handbuch der Normal und Patologische Psychologie (pp. 594-678). Berlin: Springer-Verlag.

GiBBS, T., \& LAwsON, R. B. (1974). Simultaneous brightness contrast in stereoscopic space. Vision Research, 14, 983-987.

Gilchrist, A. (1988). Lightness contrast and failures of constancy: A common explanation. Perception \& Psychophysics, 43, 415-424.

Gilchrist, A., Delman, S., \& JaCobsen, A. (1983). The classification and integration of edges as critical to the perception of reflectance and illumination. Perception \& Psychophysics, 33, 425-436.

JACOBSEN, A., \& Gilchrist, A. (1988). The ratio principle holds over a million-to-one range of illumination. Perception \& Psychophysics, $43,1-6$.

Jund, W. E., \& ARMington, J. C. (1975). Color and brightness cont rast effects as a function of spatial variables. Vision Research, 15, 917929.

KaRDOS, L. (Ed.) (1934). Ding und Schatten [Object and shadow]. [Special issue]. Zeitschrift für Psychologie, 22.

KingDOM, F., \& Moulden, B. (1991). A model for contrast discrimination with incremental and decremental test patches. Vision Research, 31, 851-858.

Lucassen, M. P., \& Walraven, J. (1993). Quantifying color constancy: Evidence for nonlinear processing of cone-specific contrast. Vision Research, 33, 739-757.

O' SHEA, R. P., \& SAvoY, R. L. (1995). Colour constancy for real and computer-simulated papers. Manuscript in preparation.

Paradiso, M. A., \& Nakayama, K. (1991). Brightness perception and filling-in. Vision Research, 31, 1221-1236.

SAVOY, R. L., \& O' SHEA, R. P. (1993). Color constancy with reflected and emitted light. Perception, 22(Suppl.), 61.

Schirillo, J., Reeves, A., \& Arend, L. E. (1990). Perceived lightness, but not brightness, of achromatic surfaces depends on perceived depth information. Perception \& Psychophysics, 48, 82-90.

Schirillo, J., \& Shevell, S. K. (1993a). Brightness contrast from a complex surround requires a complex description. Investigative Ophthalmology \& Visual Science, 34(Suppl.), 746.

Schirillo, J., \& Shevell, S. K. (1993b). Lightness and brightness judgments of coplanar retinally noncontiguous surfaces. Journal of the Optical Society of America A, 12, 2442-2452.

Shevell, S. K., Holliday, I., \& Whittle, P. (1992). Two separate neural mechanisms of brightness induction. Vision Research, 32, 2331-2340.

VAlBerG, A., \& LANGE-MaLeck1, B. (1990). "Colour constancy" in Mondrian patterns: A partial cancellation of physical chromaticity shifts by simultaneous contrast. Vision Research, 30, 371-380.

White, M. (1981). The effect of the nature of the surround on the perceived lightness of gray bars within square-wave test gratings. Perception, 10, 215-230.

WhITTLE, P. (1992). Brightness, discriminability, and the "crispening" effect. Vision Research, 32, 1493-1507.

\section{NOTE}

1. We use "articulation" as a rough measure of spatial structure and complexity in the proximal stimulus. Thus, a homogeneous field is less articulated than a bipartite field such as the background of the simultaneous lightness contrast stimulus. A bicolored regular checkerboard is less articulated than a Mondrian. Of course, our working definition is far from satisfactory. First of all, it confounds two factors, the number of edges and the number of different luminance ratios in the proximal stimulus. Intuitively, we expect these to have very different effects on lightness. Further, our working definition is structure blind. We have little doubt that the way edges and ratios are arranged is far more important than the sheer number of them. Finally, it seems intuitively necessary that articulation should take into account structure at different spatial scales.

(Manuscript received February 9, 1995; revision accepted for publication July 27, 1995.) 